\title{
The Effects of EBSD Collection Speed on Indexing Accuracy
}

\author{
M.M. Nowell, J.K. Farrer, and R.A. Witt
}

TSL/EDAX, 392 E. 12300 S., Draper, UT 84020 USA

The use of Electron Backscatter Diffraction (EBSD) and Orientation Imaging Microscopy (OIM) has become a standard technique for the microstructural analysis of crystalline materials [1]. Since its introduction a decade ago, the data collection speeds have increased by over two orders of magnitude. The latest increases have been brought about by the use of megapixel CCD cameras that produce frame rates greater than 60 frames per second (fps) [2]. These rates are achieved by using the camera in a binning mode, where several pixels on the CCD chip are combined together to create a single effective pixel. In this work, the effect of pixel binning and acquisition speed on the accuracy of OIM scans is examined.

EBSD patterns were collected from an Inconel 600 alloy, mechanically polished down to 0.05 micron colloidal silica. A Philips/FEI XL-30 FEG SEM was operated at an acceleration voltage of $20 \mathrm{kV}$ and an incident beam current of approximately $3.65 \mathrm{nA}$. EBSD patterns were obtained using a TSL DigiView 1412 camera. Four camera settings were used: A) 8x8 (174x130 pixels) binning with normal (65\% max) gain and 18.89 ms exposure, B) $8 \times 8$ binning with high (85\% max) gain and $11.66 \mathrm{~ms}$ exposure, C) $8 \times 8$ binning with no gain and $59.17 \mathrm{~ms}$ exposure, and D) 4x4 (348x260 pixels) binning with no gain and $204.08 \mathrm{~ms}$ exposure. Representative patterns at these four settings are shown in Figure 1. OIM scans of 113,825 points were collected over a $1450 \times 1087.50$ micron area with a step size of 4 microns, each from the same area. Contamination effects from previous scans were found to be negligible. At each of the four camera settings, two different Hough transform settings were used, for a total of eight scans. The first set of Hough transform settings were optimized for speed by decreasing the transform resolution to 2 degrees in theta and searching for 6 lines. The second set of Hough settings were set by increasing the resolution to 0.5 degrees in theta and searching for 8 lines.

To evaluate the accuracy of the OIM scan results, the confidence index (CI) distribution, grain average misorientation, and grain orientation spread were examined. The average time per point was also recorded. These results are shown in Table 1. CI values greater than 0.1 indicate correct orientations at least $95 \%$ of the time [3]. A CI standardization procedure was also used to identify points with the correct orientation but a CI value less than 0.1 . In this procedure, a grain boundary tolerance angle is set (typically to 5 degrees), and orientation measurements are grouped into grains with no point-to-point misorientations greater than the tolerance angle. The highest CI value within each grain is then assigned to all other points within the grain. More than $99.5 \%$ of the points were indexed correctly, regardless of camera and Hough settings. The grain average misorientation is the average misorientation between all neighboring pairs of points within a grain. The grain orientation spread is determined by calculating the misorientation between all points within a grain. The orientation spread is the average misorientation value. For comparison, OIM scans were collected over the same sized area from s single crystal silicon specimen while varying the camera and Hough settings as above. These results are given in Table 2. While lowering the gain does improve the precision of the misorientation measurements slightly, the grain average misorientations were all less 
than 0.9 degrees. Figure 2 shows the spatial location of the low CI points. These points are mostly located at scratches, grain boundaries, and artifacts on the surface.

[1] A.J. Schwartz et al., Electron Backscatter Diffraction in Materials Science, Kluwer Academic/Plenum Publishers, New York, 2000.

[2] D.J. Dingley et al., Microsc. Today. 3 (2002) 10-14.

[3] D.P. Field,Ultramicroscopy. 67 (1997) 1-9.

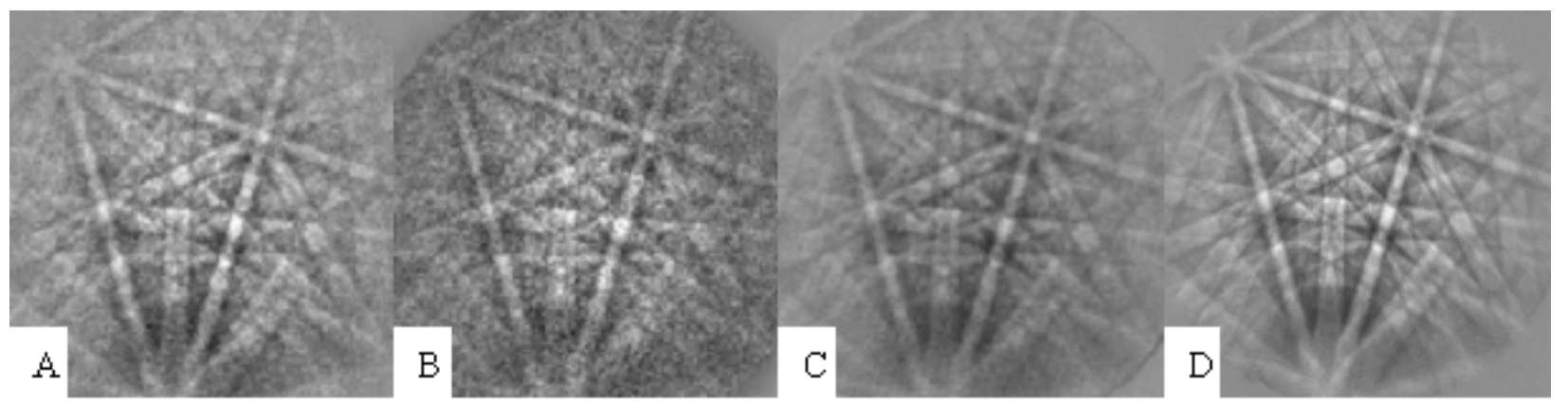

FIG. 1.EBSD Patterns collected with camera settings at: A) 8x8 binning at normal gain, B) 8x8 binning at high gain, C) $8 \times 8$ binning at no gain, D) $4 \times 4$ binning at no gain.

TABLE 1. Confidence Index, misorientation values (in degrees), and points per second at varying scan settings.

\begin{tabular}{lcccccccc}
\hline Camera Setting & A & A & B & B & C & C & D & D \\
Hough Setting & 1 & 2 & 1 & 2 & 1 & 2 & 1 & 2 \\
\hline \% CI $>$ 0.1 Before CI Standardization & 98.9 & 99.0 & 98.8 & 98.8 & 98.7 & 98.7 & 98.8 & 99.3 \\
\% CI $>$ 0.1 After CI Standardization & 99.6 & 99.8 & 99.6 & 99.7 & 99.6 & 99.8 & 99.7 & 99.8 \\
\hline Average Misorientation & 0.80 & 0.83 & 0.89 & 0.84 & 0.76 & 0.70 & 0.82 & 0.74 \\
Orientation Spread & 1.16 & 1.16 & 1.19 & 1.18 & 1.14 & 1.11 & 1.18 & 1.13 \\
\hline Points per Second & 68 & 16.8 & 68 & 16.8 & 16.8 & 16.8 & 4.9 & 4.9
\end{tabular}

TABLE 2. Misorientation values for single crystal silicon scans at varying settings. Values are in degrees.

\begin{tabular}{lllllllll}
\hline Camera Setting & A & A & B & B & C & C & D & D \\
Hough Setting & 1 & 2 & 1 & 1 & 1 & 2 & 1 & 2 \\
\hline Average Misorientation & 0.27 & 0.20 & 0.32 & 0.24 & 0.18 & 0.13 & 0.21 & 0.15 \\
Orientation Spread & 0.42 & 0.32 & 0.45 & 0.33 & 0.38 & 0.29 & 0.47 & 0.29
\end{tabular}
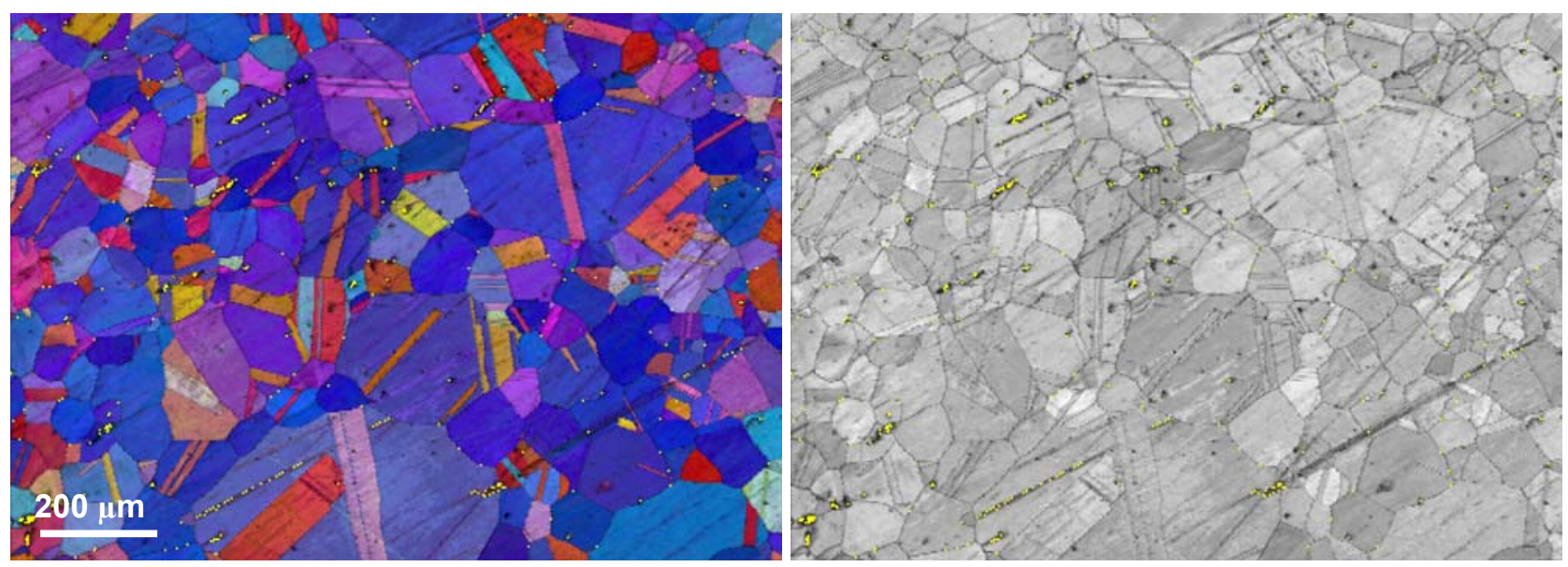

Fig. 2. Orientation and Image Quality maps, acquired at settings A-1, showing the locations of low CI points as yellow points. These points are primarily located at scratches, grain boundaries, and artifacts. 